\title{
Investigation of Rate of Photo Degradation of Chlorothalonil, Lambda Cyhalothrin, Pentachlorophenol and Chlropysis on Tomato and Spinach
}

\author{
Mbugua JK*, Mbui D and Kamau GN
}

University of Nairobi, Nairobi, Kenya

\begin{abstract}
Photo-degradation of common pesticides and herbicides on the surface of tomato fruit and spinach leaves were studied. Samples were spiked with $100 \mathrm{mg} / \mathrm{ml}$ of lambda cyhalothrin, chlorothalonil, chlorpyriphos and pentachlorophenol standard solutions in acetone. Thy were air dried for 1 minute and exposed to light of various intensities; sunlight, $40 \mathrm{w}, 60 \mathrm{w}, 75 \mathrm{w}$ and $100 \mathrm{w}$ bulbs after spreading on the surface of spinach and tomatoes for 15, 30,45 and 60 minutes and then washed in acetone. The pesticide concentration in the samples was analyzed with a UV Visible spectrophotometer. The rate of degradation of chlorothalonil, lambda cyhalothrin, pentachlorophenol and chlorpyriphos was also calculated and rate constants obtained for each residue. The results obtained indicated that the $100 \mathrm{~W}$ bulb degradation ranged from $20-95 \%$ for all the molecules in both tomato fruit and spinach leave surface. The residues breakdown followed a $1^{\text {st }}$ order kinetics.
\end{abstract}

Keywords: Residue; Photo-degradation; Chlorothalonil; Dursban; Lambda cyhalothrin; Pentachlorophenol; Spectrophotometry

\section{Introduction}

Integrated pest management (IPM) strategies employ the use of different classes of pesticides on agricultural crops. Frequent use of pest control chemicals affects the properties of the soil and ground water by altering the $\mathrm{pH}$ thereby inhibiting microbial activities which is important for soil fertility $[1,2]$. Approximately $0.1 \%$ of applied pesticides reach the target pest, which means that the bigger percentage of the pesticide ends up contaminating the environment [3]. Photo-degradation is an abiotic process in the dissipation of residues where molecular excitation by absorption of photons result in organic reaction [4]. The degradation of pesticides is mainly focused on hydrolysis, photo-degradation, and microbial degradation [4-6]. Photo-degradation of pesticides has a significant influence on pesticide residue, efficacy and toxicity and in a big way determines the fate of pesticides on the crop surface and in the atmospheric and water environment [5]. The photochemical degradation properties of pesticides have become an important index in ecological environment safety evaluation of pesticide. Photo-degradation study is required to provide information of photo-degradation for pesticide registration in many countries. Organo-phosphates are normally stable at ambient temperatures and their isomers are formed at elevated temperatures. For example, the $\mathrm{P}=\mathrm{S}$ (thiono) bond where $\mathrm{P}$ is phosphate isomerizes to $\mathrm{P}=\mathrm{S}$ (thiolo) which are more toxic to mammals [7-9]. Ultra-violet light is a high source of energy, and promotes photodegradation. Some pesticides are equipped with UV light blockers so as to reduce the rate of photo decomposition of their active ingredient [10]. Since the UV-violet component of sunlight which varies from 200-400 nm degrades the residues, it is vital to study the effect of light on degradation of pesticide molecules to understand their fate in the environment when applied $[11,12]$.

\section{Materials}

All chemicals used were of analytical grade quality $99.8 \%$ Acetone, 99.8\% Acetonitrile and obtained from 64271 Darmstadt, Germany and Aldrich respectively; Distilled water was double distilled into a glass bottle; 99.3\% Pentachlorophenol, 99.8\% Chlorpyrifos and 99.3\% lambda cyhalothrin from labor Dr. Ehrenstorfer; $99.0 \%$ Chlorothalonil from Annopol 6 Warsaw Poland, 1\% Acetic Acid HPLC grade from Sigma Adrich. UV-Visible spectrophotometer (Shimadzu UV-Visible 1650 PC Shimadzu Scientific Instruments, 7102 River wood Drive Columbia, MD 21046, USA), Analytical balance (Fischer scientific A-160); $1 \mathrm{~cm}$ quartz cuvette.

\section{Methodology}

\section{Stock solution preparation}

$1000 \mathrm{ppm}$ stock solution for chlorothalonil, chlorpyrifos and pentachlorophenol were prepared in acetone while distilled water was used for lambda cyhalothrin. 10, 20, 40, 60, 80 and $100 \mathrm{mg} / \mathrm{liter}$ solutions were prepared by serial dilution method. The solutions were scanned using UV-Visible spectrophotometer. Plot of absorbance versus concentration for each pesticide standard was made and further used in the degradation study. Five sets of spinach leaves $(5 \mathrm{~cm}$ wide) and tomatoes were placed in a separate petri dishes. $2 \mathrm{ml}$ each of the acetone-dissolved $1000 \mathrm{mg} / \mathrm{l}$ stock solutions was spread on the plant surface and the acetone allowed to evaporate for 1 minute. The set was then exposed to $40 \mathrm{w}, 60 \mathrm{w}, 75 \mathrm{w}$ and $100 \mathrm{w}$ bulbs which were enclosed in a container to prevent light loss for 4 hours. Another set up was exposed to sunlight for 4 hours. Temperature in each set up was recorded during the exposure. The samples were then allowed to stabilize in the laboratory for 24 hours before washing with $10 \mathrm{ml}$ acetone. The extracts were analyzed for the pesticide levels using UVVisible spectrometer at $340 \mathrm{~nm}$ for chlorothalonil, chlorpyriphos and pentachlorophenol and $254 \mathrm{~nm}$ for lambda cyhalothrin. The amount of residue left after exposure was determined. To study the effect of time on the rate of degradation of these pesticide residues, $5 \mathrm{~cm}$ by $5 \mathrm{~cm}$ of spinach leaves were cut and dipped in $100 \mathrm{ppm}$ standard solutions of chlorothalonil, chlorpyriphos, pentachlorophenol and lambda cyhalothrin prepared in acetone for 2 minutes. The leaves were placed in a Petri dish and acetone allowed to evaporate in air for 1 minute. The setups in the petri dishes were then were exposed to $40 \mathrm{w}, 60 \mathrm{w}$, $75 \mathrm{w}$ and $100 \mathrm{w}$ bulbs for 10, 20, 30 and 60 minutes each. The spiked samples were removed and washed with $5 \mathrm{ml}$ acetone and then residue concentration analyzed with UV-Visible spectrophotometer.

*Corresponding author: Mbugua JK, University of Nairobi, Nairobi, Kenya, Tel: +254724305124; E-mail: djames085@gmail.com

Received December 01, 2016; Accepted December 15, 2016; Published January 02, 2017

Citation: Mbugua JK, Mbui D, Kamau GN (2017) Investigation of Rate of Photo Degradation of Chlorothalonil, Lambda Cyhalothrin, Pentachlorophenol and Chlropysis on Tomato and Spinach. Mod Chem Appl 5: 200. doi: 10.4172/23296798.1000200

Copyright: ( $) 2017$ Mbugua JK, et al. This is an open-access article distributed under the terms of the Creative Commons Attribution License, which permits unrestricted use, distribution, and reproduction in any medium, provided the original author and source are credited. 


\section{Kinetic study}

In order to study the rate of degradation of chlorpyriphos, pentachlorophenol, lambda cyhalothrin and chlorothalonil formulations plots of time versus natural logarithm of concentration were made to find the rate of degradation while the half lives period $\left(\mathrm{t}_{0.5}\right)$ were calculated according to equation [13].

$$
\mathrm{t}_{0.5}=\ln 2 / \mathrm{k}=0.693 / \mathrm{k}
$$

\section{$\mathrm{k}=1 / \mathrm{Tx} \cdot \ln \mathrm{a} / \mathrm{bx}$}

where, $\mathrm{k}=$ Rate constant, $\mathrm{T}_{\mathrm{x}}=$ Time in days, $\mathrm{a}=$ Initial residue, $\mathrm{b}_{\mathrm{x}}=$ Residue at time $(\mathrm{x})$.

\section{Results and Discussion}

The plots of absorbance against concentration were observed to adhere to Beer's law with regression values of $0.969,0.981,0.973$ and 0.992 for lambda cyhalothrin, pentachlorophenol, chlorothalonil and chlorpyriphos, respectively confirming linearity of the obtained absorbance. Table 1 below show the calibration curves equations with their regression values for the four pesticides.

\section{The rate of degradation}

The decrease in pesticides residues concentrations as depicted in Figures 1a-1d confirms the degradation of chlorpyriphos, lambda cyhalothrin, chlorothalonil and pentachlorophenol on exposure to different light intensities on the surface of the spinach leaf. For all the four residues under study, photo degradation is observed to be dependent on time of exposure, light intensity and temperature. The percentage loss is higher in pentachlorophenol, chlorothalonil with chlorpyriphos and lambda cyhalothrin degrading almost at the same rate at $100 \mathrm{w}$ on exposure for 60 minutes. The $\%$ loss for $100 \mathrm{w}$ on exposure for 60 minutes is $87,83,71$ and 71 for Pentachlorophenol, chlorothalonil, lambda cyhalothrin and chlorpyriphos, respectively. The difference in the loss is most probably due to the difference in molecular structure. This corroborates results given were observed that photo degradation of organic molecules is highly influence by the molecular structure. To determine the rate constant of residues, plots of natural logarithm of concentration against time were made. The rate constant is obtained from slope of the plots (Figure 2). The plots confirm that the reactions are first order as expected.

\begin{tabular}{|c|c|c|}
\hline Pesticide & Equation & Regression \\
\hline Chlorpyriphos & $\mathrm{Y}=0.001 \mathrm{x}+0.003$ & 0.992 \\
\hline Pentachlorophenol & $\mathrm{Y}=0.004+0.009$ & 0.981 \\
\hline Chlorothalonil & $\mathrm{Y}=0.005 \mathrm{x}+0.006$ & 0.993 \\
\hline Lambda cyhalothrin & $\mathrm{Y}=0.010+0.015$ & 0.969 \\
\hline
\end{tabular}

Table 1: Calibration curves equation.

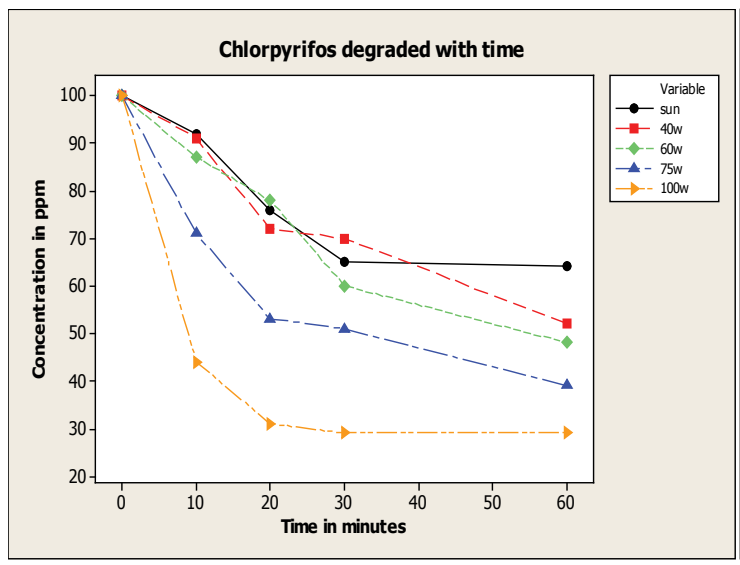

a

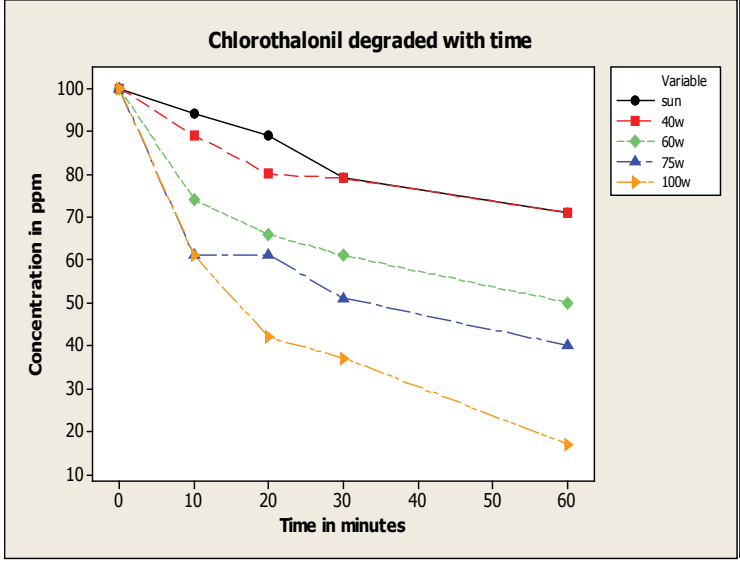

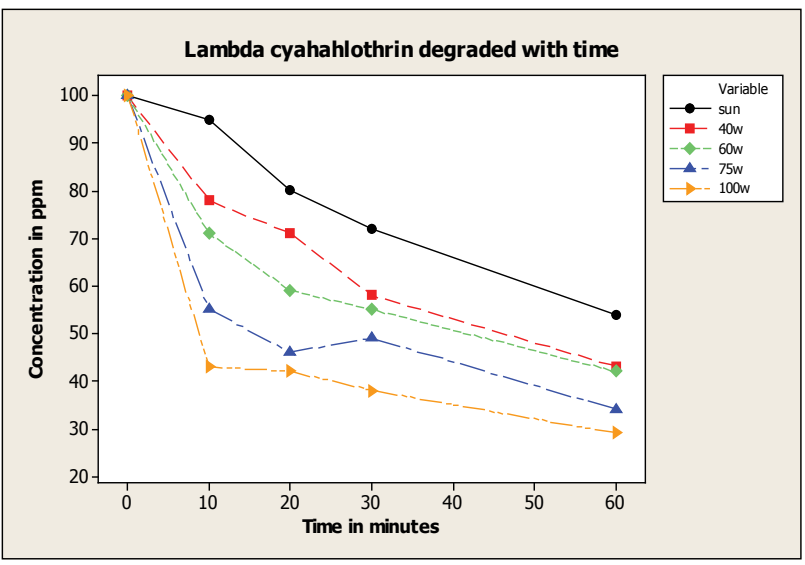

b

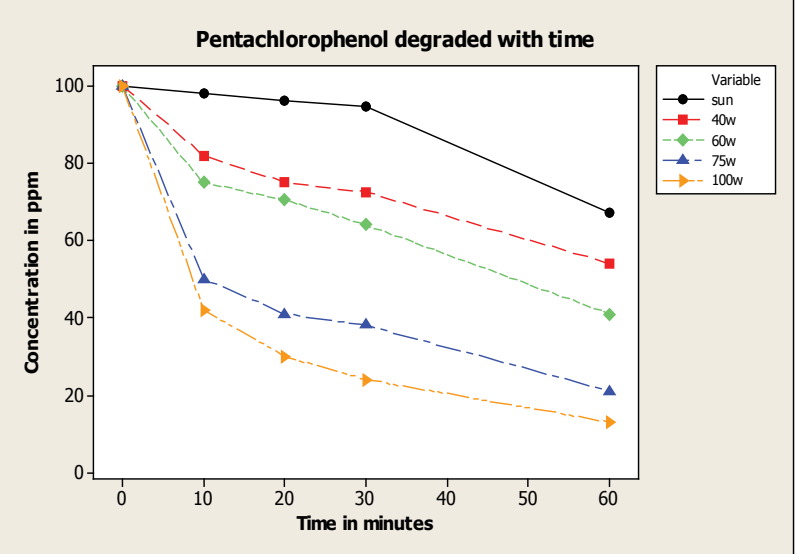

d

Figures 1: Photodegradation trend of (a) chlorpyrifos (b) lambda cyhalothrin (c) chlorothalonil (d) lambda cyhalothrin with time in minutes. 

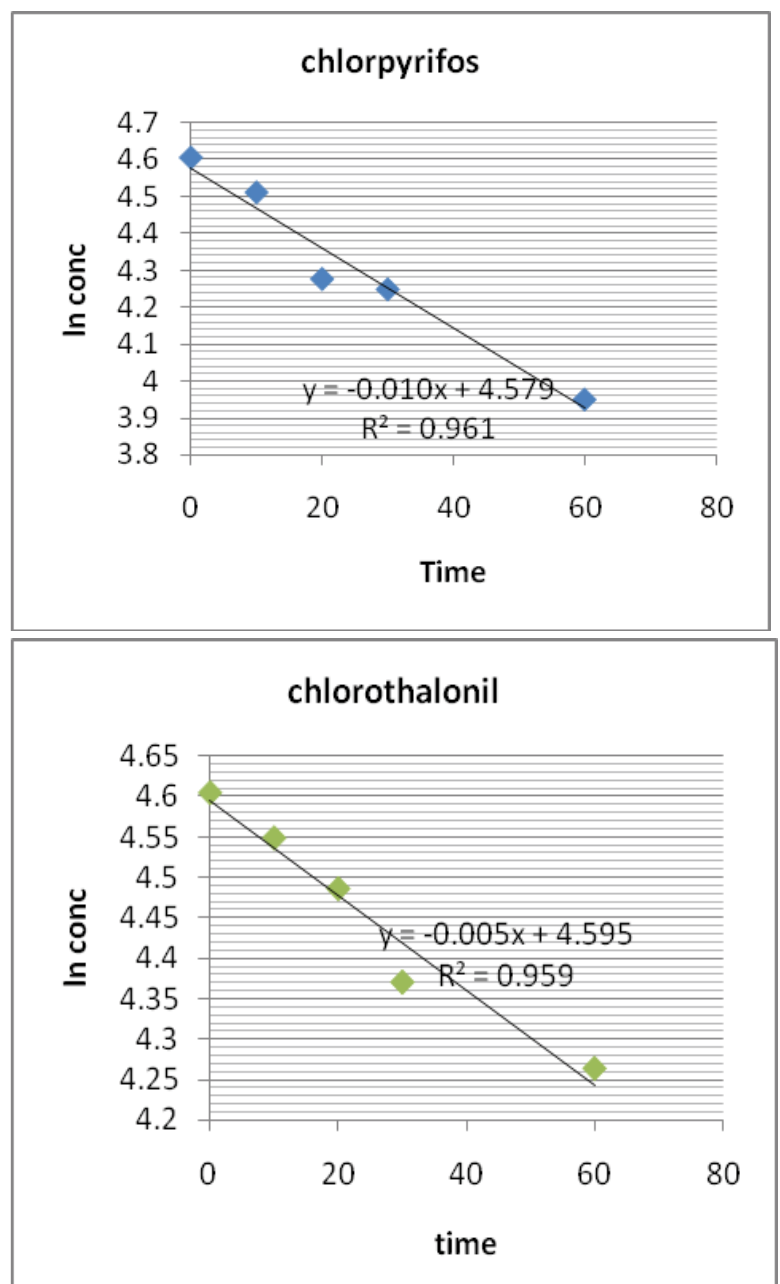

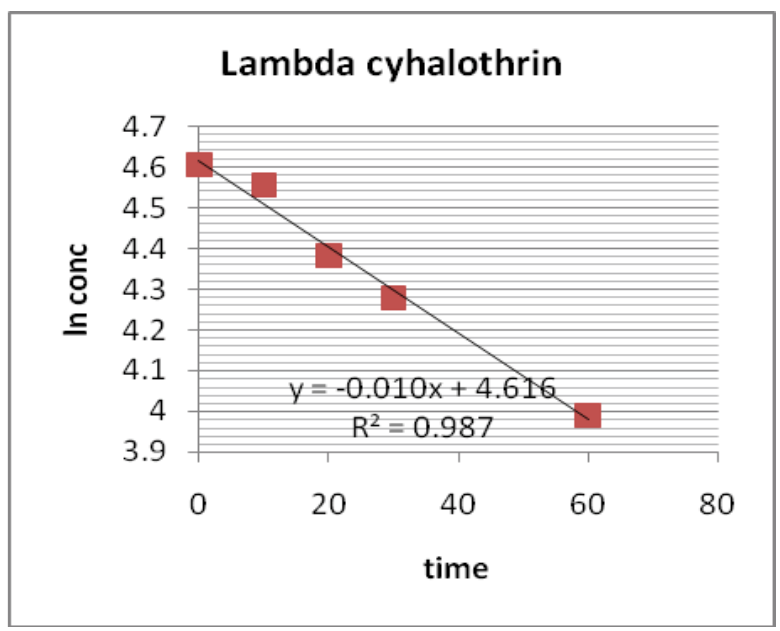

\section{Pentachlorophenol}

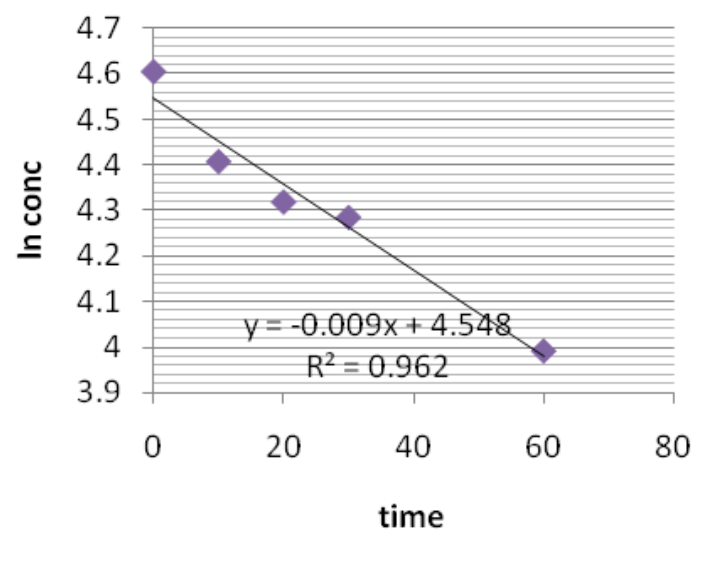

Figures 2: Plots of In concentration versus time for pesticide residues for $100 \mathrm{~W}$ bulb.

\section{Energy calculations}

The total amount of energy absorbed by $5 \mathrm{~cm}$ by $5 \mathrm{~cm}$ leaf surface from the sun on a hot summer can be calculated as follows; Energy=power $\times$ time. Electromagnetic wave from the sun is $1.4 \mathrm{~kW} / \mathrm{m}^{2}$ but only $80 \%$ of this reaches the earth surface on a hot summer day, therefore, $80 / 100 \times 1.4=1.12 \mathrm{~kW} / \mathrm{m}^{2}$; Area $=5 \mathrm{~cm} \times 5 \mathrm{~cm}=25 / 10000=0.0025 \mathrm{~m}^{2}$ power $=0.0025 \mathrm{~m}^{2} \times 1.12 \mathrm{~kW} / \mathrm{m}^{2}=0.0028 \mathrm{~kW}$, Energy $=0.0028 \mathrm{~kW} \times$ $14400 \mathrm{sec}=40.32$ joules. This means that the amount of energy absorbed by the spinach and tomato when exposed to $40 \mathrm{w}, 60 \mathrm{w}, 75 \mathrm{w}$ and 100 $\mathrm{w}$ bulbs for four hours can be calculated since the surface area and the wattage is known. The amount of energy responsible for breaking down the pesticide molecules are shown in Table 2. The sun has the highest amount of energy reaching the surface of the 0.0025 and $0.002798 \mathrm{~m}^{2}$ of the spinach and tomato respectively. From the plots of percentage degradation of the four residues, the sun degraded the least. Several factors account for this degradation trend; the distance from the sun to the molecule surface is $93,000,000$ miles meaning the intensity

\begin{tabular}{|c|c|c|}
\hline Light & Spinach $\left(\mathrm{t}_{1 / 2}\right)$ & Tomatoes $\left(\mathrm{t}_{1 / 2}\right)$ \\
\hline $40 \mathrm{w}$ & 0.060 & 0.059 \\
\hline $60 \mathrm{w}$ & 0.058 & 0.058 \\
\hline $75 \mathrm{w}$ & 0.055 & 0.055 \\
\hline $100 \mathrm{w}$ & 0.045 & 0.054 \\
\hline
\end{tabular}

Table 2: Half-lives of lambda cyhalothrin. reaching the crop surface is reduced to a large extent unlike for the bulb which is $30 \mathrm{~cm}$ above the surface. Secondly, as noted earlier by Suett temperature is a major factor in the breakdown of pesticides whereby he reported doubling of chemical reactions with $10^{\circ} \mathrm{C}$ rises in temperature. Photo-degradation from the sun is influence by light intensity while light intensity and temperature influences breakdown of residues. This explains the pattern of photo-degradation (Figure 3).

\section{Lambda cyhalothrin}

Exposure of lambda-cyhalothrin spiked spinach and tomatoes samples to sunlight, bulb light of $40 \mathrm{w}, 60 \mathrm{w}, 75 \mathrm{w}$ and $100 \mathrm{~W}$ ) for four hours resulted in nearly complete degradation of cyhalothrin with losses greater than $95 \%$ of initial amounts applied (Figure 4). The degradation was observed to vary with light intensity. Different wattage bulbs emitted different amount of light intensity. This means that photodegradation of lambda cyhalothrin on the surface of spinach; tomato and blank petri dish resulted from effects of light intensity. Figure 5 illustrate the amount degraded versus wattage. Lambda cyhalothrin degradation was highest in $100 \mathrm{~W}$ bulb as expected from light. This is in agreement with the Stark Einstein law which stipulates that for every photon absorbed a molecule undergoes photochemical reactions. This means that the reaction depends on the number of photons that illuminate a surface (wattage) and the bond being cleaved (molecular structure) in the two plants investigated the half life decreases with the strength of radiation which further agrees with Stark Einstein law; the 


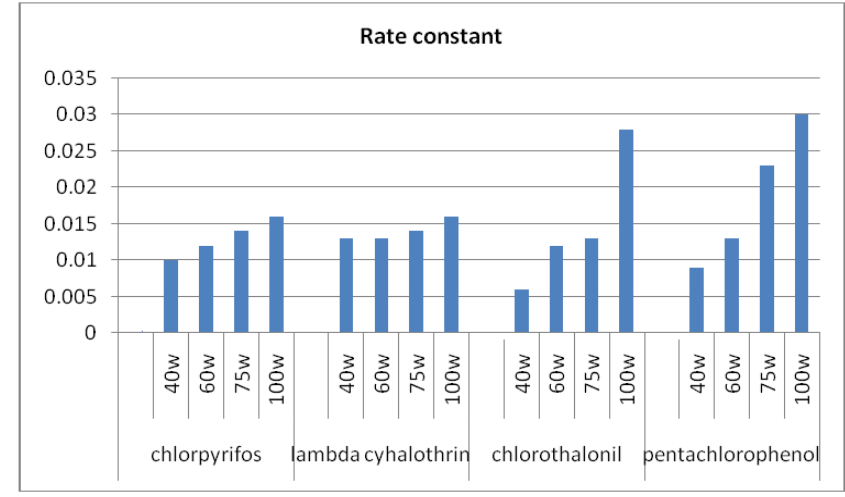

Figure 3: Plot of rate constant of pesticide residues on spinach.

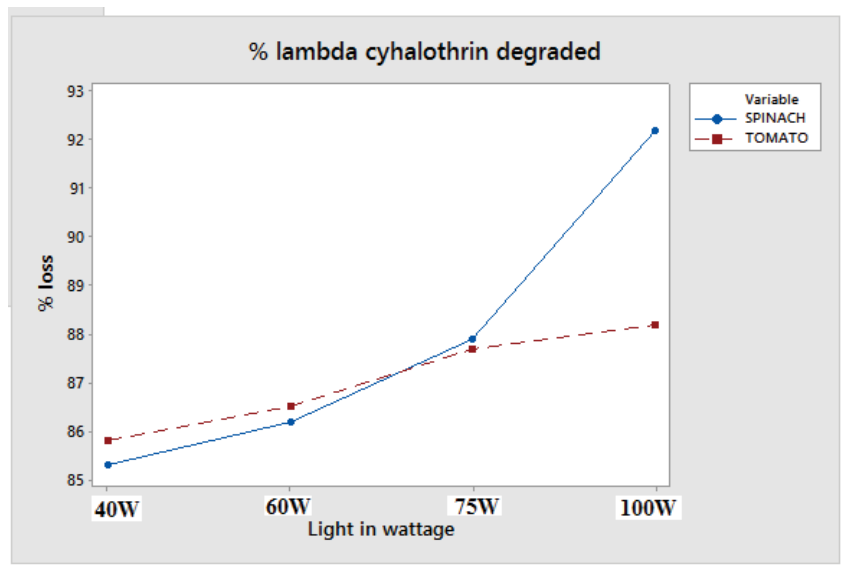

Figure 4: \% lambda cyhalothrin degraded with respect to light.

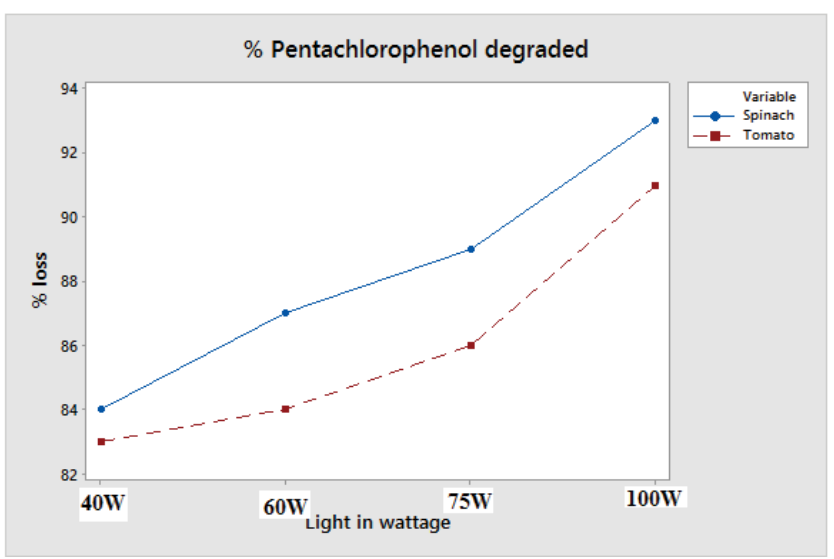

Figure 5: \% degraded pentachlorophenol against light.

higher the radiant energy, the higher the number of photons involved, and the higher the amount of molecules undergoing reaction and therefore the shorter the amount of time required for half the entities to photo-degrade. The results obtained for lambda cyhalothrin photodegradation are consistent with those earlier observed they found that on exposure of Lambda cyhalothrin to UV-light $(18 \mathrm{~W}, 254 \mathrm{~nm})$ for 20 minutes resulted to more than $95 \%$ loss of the initial concentrations. The protective waxy coating in tomatoes explain the lower degradation of this molecule on the tomato surface. The degradation followed a first order kinetics with an average half-life of 0.0545 on spinach leaf surface and 0.0565 on tomato surface. Similar results were observed by Fernandez who obtained a half-life of 4.26 min for lambda cyhalothrin degradation by UV-light. Table 3 below indicate the obtained half-life on exposure of the molecule for 4 hours to different light intensities. Except for the 100 wattage, the half-lives do not seem to depend on the species of the plant under investigation, which may imply that at relatively low wattage, reaction rate only depends on the radiant energy and the temperature $[14,15]$.

\section{Pentachlorophenol}

When Pentachlorophenol was exposed to different energy bulbs, the amount of Pentachlorophenol after 4 hours was obtained and the trend of the residues degraded against light (Figure 6) is shown below. As earlier observed for lambda cyhalothrin, the loss of Pentachlorophenol on radiating with light of different intensities was dependent on wattage. The percentage loss was lowest for $40 \mathrm{~W}$ bull and is explained by the fact that the number of photons is less compared to $100 \mathrm{~W}$ bulb. This means that the number of molecules undergoing photo-degradation is less compared to $60 \mathrm{~W}, 75 \mathrm{~W}$ and $100 \mathrm{~W}$ cases. The half-lives for spinach are consistently higher than those of tomatoes. It could be that there are two competing reactions which mean that less Pentachlorophenol is degraded per unit time i.e., the rate of photodegradation of Pentachlorophenol is lower and thus takes much longer to get to half the initial concentration. The protective coat and lack of chlorophyll in tomato make the half-life less than that of spinach. It is worth noting that half-lives of Pentachlorophenol are much higher than those of lambda cyhalothrin at all instances, which may be due to the structure of Pentachlorophenol (Table 4).

\begin{tabular}{|c|c|c|}
\hline Light & Spinach $\left(\mathbf{t}_{\mathbf{1 / 2}}\right)$ & Tomatoes $\left(\mathbf{t}_{\mathbf{1 / 2}}\right)$ \\
\hline $40 \mathrm{w}$ & 0.141 & 0.115 \\
\hline $60 \mathrm{w}$ & 0.105 & 0.096 \\
\hline $75 \mathrm{w}$ & 0.091 & 0.089 \\
\hline $100 \mathrm{w}$ & 0.060 & 0.055 \\
\hline
\end{tabular}

Table 3: Half-lives of pentachlorophenol on spinach and tomato.

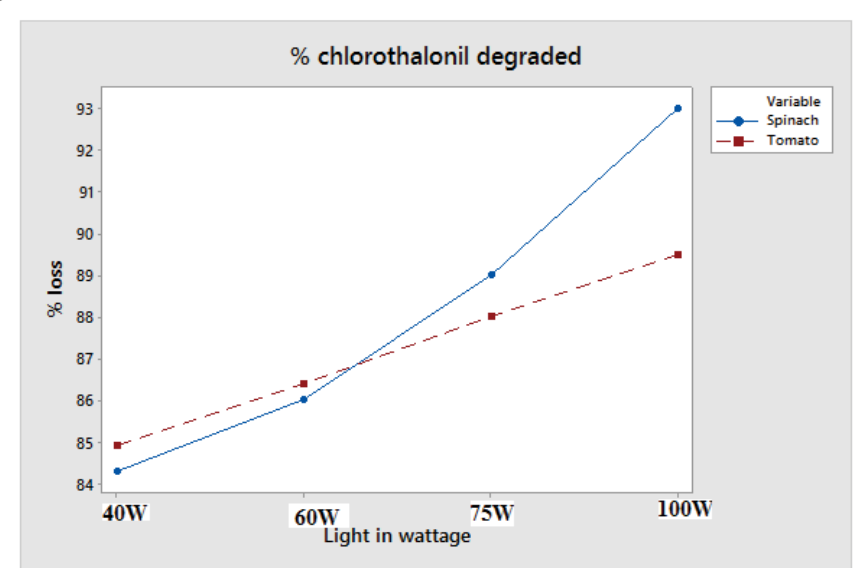

Figure 6: \% Degraded of chlorothalonil after four hours of exposure to different lights.

\begin{tabular}{|c|c|c|}
\hline Light & spinach & tomatoes \\
\hline $40 \mathrm{w}$ & 0.079 & 0.080 \\
\hline $60 \mathrm{w}$ & 0.058 & 0.058 \\
\hline $75 \mathrm{w}$ & 0.054 & 0.054 \\
\hline $100 \mathrm{w}$ & 0.041 & 0.041 \\
\hline
\end{tabular}

Table 4: Calculated half-lives of chlorothalonil. 


\section{Chlorothalonil}

Chlorothalonil is an organochlorine molecule. Just like in case of Pentachlorophenol, the \% loss of chlorothalonil on exposure to lights of varying intensities was depended on light intensity as shown in Figure 7. Like lambda cyalothrin the photo degradation of chlorothalonil is found to be independent of species of crop analysed, but only dependent on wattage which could indicate that they have similar photo-degradation patterns. The presence of the cyano group in both species may greatly influence the degradation pattern in the plants in the investigated. Despite the fact that the half-life obtained are comparable to those of lambda cyhalothrin, the amount of chlorothalonil degraded by each of the radiated lights is much lower than that of lambda cyhalothrin (Figure 8). This can be due to the amount of energy required to break of the $\mathrm{Cl}-\mathrm{C}$ (in benzene) bond $(339 \mathrm{KJ} / \mathrm{mol})$; thus eventually this is the amount of energy required to degrade the pesticide even with the same amount of radiation. Once more the half-lives were observed to decreases with wattage due to increased number of photons degrading more molecules (Tables 5 and 6 ).

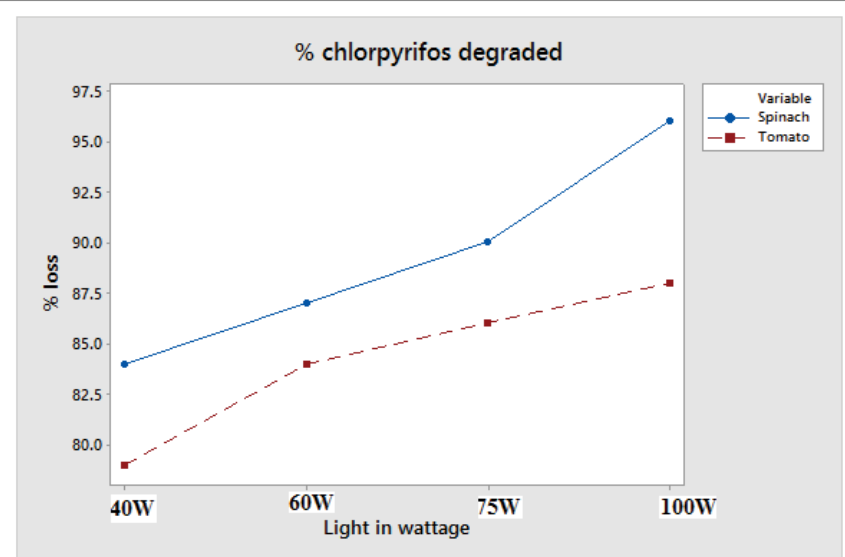

Figure 7: Amount of chlorpyriphos lost after four hours of exposure to different lights.

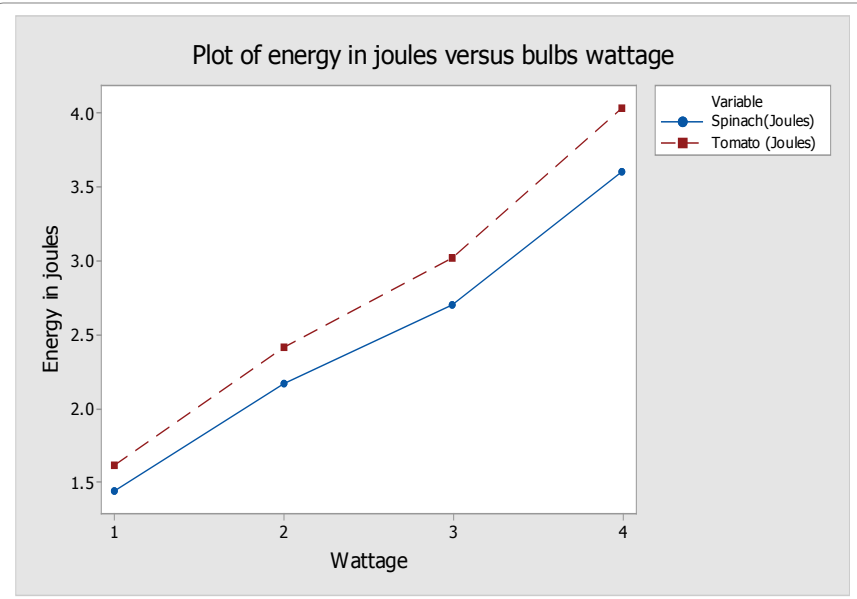

Figure 8: Plot of energy in joules versus (a) light and (b) temperature.

\begin{tabular}{|c|c|c|}
\hline Light & spinach $\left(\mathbf{t}_{1 / 2}\right)$ & tomatoes $\left(\mathbf{t}_{\mathbf{1 / 2}}\right)$ \\
\hline $40 \mathrm{w}$ & 0.065 & 0.065 \\
\hline $60 \mathrm{w}$ & 0.064 & 0.064 \\
\hline $75 \mathrm{w}$ & 0.062 & 0.062 \\
\hline $100 \mathrm{w}$ & 0.042 & 0.042 \\
\hline
\end{tabular}

Table 5: Calculated half-lives of chlorpyriphos.

\begin{tabular}{|c|c|c|}
\hline Light & Spinach (Joules) & Tomato (Joules) \\
\hline Sunlight & 40.3200 & 45.0901 \\
\hline $40 \mathrm{w}$ & 1.4400 & 1.6105 \\
\hline $60 \mathrm{w}$ & 2.1600 & 2.4157 \\
\hline $75 \mathrm{w}$ & 2.7000 & 3.0197 \\
\hline $100 \mathrm{w}$ & 3.6000 & 4.0263 \\
\hline
\end{tabular}

Table 6: Energy reaching the plant surface.

\section{Chlorpyriphos}

Chlorpyriphos is an organophosphate insecticide whose degradation behavior is highly dependent on light intensity and surface of exposure as indicated in Figure 8 below. Like lambda cyhalothrin and chlorothalonil, photodegradation of chlorpyriphos is independent of species of crops analysed but dependent on light radiated. This indicates that the photo degradation mechanism of chlorpyriphos on spinach and tomatoes are similar. Contrary to that, irradiation on blank surface indicates that the half-life of chlorpyriphos is higher than that on the spinach and tomatoes. The half-lives were observed to decrease with wattage due to the photons degrading more molecules.

\section{Conclusion}

Photo-degradation of residues on the surface of spinach leaf by different light intensity followed first order kinetic. The degradation rate was in sequence of $100 \mathrm{w}>75 \mathrm{w}>60>40>$ sun. The rate of degradation heavily relied on temperature, exposure time and light intensity. The half-life ranged from 0.069 to 0.141 days for spinach and $0.074-0.105$ days for tomatoes. The rate of photo-degradation was heavily dependent on the molecular structure of the residue. Pesticide administration on vegetables and other fast growing crops should be done cautiously to avoid food contamination. The by-products of residues photodegradation may be more hazardous than the parent molecules. This study therefore recommends thorough washing of food stuff prior to use and further research in analysis the structures of photo-degradation by-products.

\section{References}

1. Rehman GKMM, Motoyama N (2000) Determination of Chlorpyriphos residue in andosol upland soils using methanol phosphoric acid extraction. J Pesticide Sci 25: 387-391.

2. Malinowski H (2000) Impact of more important factors on the activity of insecticide used in forest protection. I Soil Insecticides 144: 89-100.

3. Ardley JH (1999) Pesticide considerations on environment concern. Agricultura Sciences 12: 21-24.

4. Katagi T (2004) Photodegradation of pesticides on plant and soil surface. Rev Environ Contam Toxicol 182: 1-189.

5. Hong P, Zeng $Y$ (2002) Degradation of pentachlorophenol by ozonation and biodegradability of intermediates. Water Res 36: 4243-4254.

6. Barceló D, Durand B (1993) Photodegradation of the organophosphorus pesticides chlorpyriphos, fenamiphos, and vamidothion in water. Toxicol Environ Chem 38: 183-199.

7. Jong Hyang K, ByoungChul M (1997) Decomposition of organo phosphorous compounds with ultraviolet energy. J of Korean Ind and Eng Chemistry 9: 28-32.

8. Kuperberg JM, Soliman KFA, Stino FJR, Kolta MG (2000) Effects of time of day on chlorpyrifos induced alterations in body temperature. Life Sci 67: 2001-2009.

9. Jack R, Plimmer (2001) Handbook of Pesticide Toxicology. Academic Press, pp: 95-107.

10. Kansas State University Agricultural Experiment Station and Cooperative Extension Service (1990) Factors affecting pesticide behavior and breakdown formulations.

11. Ruzo LO, Casida JE (1982) Pyrethroid photochemistry: intramolecular sensitization and photoreactvity of 3-phenoxybenzyl, 3-phenyl-benzyl and 3-esters. J Agric Food chem 30: 963-966. 
Citation: Mbugua JK, Mbui D, Kamau GN (2017) Investigation of Rate of Photo Degradation of Chlorothalonil, Lambda Cyhalothrin, Pentachlorophenol and Chlropysis on Tomato and Spinach. Mod Chem Appl 5: 200. doi: 10.4172/2329-6798.1000200

12. James JP, Spence JH, Ford JH (1984) Gas chromatographic determination of chlorpyriphos residues in greenhouse vegetables after treatment of potting media with Dursban for imported fire ant. JAOAC 67: 1091-1094.

13. Weerasinghe CA, Matheews JM, Wright RS, Wang RY (1992) Aquatic photo degradation of Albendazole and its major metabolites.2. Reaction quantum yield, photolysis rate, and half-life in the environment. J Agric Food Chem 40: 419-421.
14. Ola MY, Emara S, Aziz AAE (2010) Effect of Heat direct Sunlight and UV-Rays on the Stability of some Chlropyrifos Formulations with Emphasis to their Content of Sulfotep. Nature and Science 8: 229-234.

15. Fernandez AM, Sanchez PL, Lores ML, Lompart M (2007) Alternative sample preparation method for photochemical studies based on solid phase microextraction: synthetic pyrethroid photochemistry. J Chromatography Sample Prep 1152: 156-167. 